\title{
Quantum Logic Network for Probabilistic Teleportation of Two-Particle State of General Form*
}

\author{
GAO Ting ${ }^{1,2}$, WANG Zhi-xi ${ }^{1}$, YAN Feng-li3 ${ }^{3,4}$ \\ ${ }^{1}$ Department of Mathematics, Capital Normal University, Beijing 100037, China \\ ${ }^{2}$ College of Mathematics and Information Science, Hebei Normal University, Shijiazhuang 050016, China \\ ${ }^{3}$ Department of Physics, Hebei Normal University, Shijiazhuang 050016, China \\ ${ }^{4}$ CCAST (World Laboratory), P.O. Box 8730, Beijing 100080, China
}

\begin{abstract}
A simplification scheme of probabilistic teleportation of two-particle state of general form is given. By means of the primitive operations consisting of single-qubit gates, two-qubit controlled-not gates, Von Neumann measurement and classically controlled operations, we construct an efficient quantum logical network for implementing the new scheme of probabilistic teleportation of a two-particle state of general form.
\end{abstract}

PACS: 03.65.Bz, 03.67.Hk

Quantum teleportation is one of the most striking progress of quantum information theory. ${ }^{1}$ It will be useful in quantum computers. ${ }^{2,3}$ It can be used to transmit information reliably in noisy situations where a message would otherwise be degraded, and to transfer information from fleeting or hard-to-control carriers to particles more suitably for permanent storage. Moreover, it may have application in quantum cryptography and quantum dense coding. ${ }^{4-8}$

In 1993, Bennett et al presented the theory of quantum teleportation which allows the transmission of an unknown qubit state from a sender "Alice" to a spatially distant receiver "Bob" via a quantum channel with the aid of some classical communication. ${ }^{1}$ Teleportation has drawn much attention because its fresh notion and latent applied prospects in quantum communication and quantum calculation. At present, teleportation has been generalized to many cases ${ }^{9-15}$ and demonstrated with the polarization photon ${ }^{16}$ and a single coherent mode of field ${ }^{17}$ in the experiments. ${ }^{18,19}$ Recently, Shi et al and Lu et al studied probabilistic teleportation of twoparticle entangled state via a three-particle nonmaximally entangled state. ${ }^{12,14}$ However, the form of two-particle entangled state is not general form of two-particle state. In order to conquer this

*Supported by National Natural Science Foundation of China under Grant No. 10271081 and Hebei Natural Science Foundation under Grant No. 101094. 
limitation, Yan et al have generalized Shi's method to the two-particle state of general form by using partly pure entangled four-particle state as the quantum channel. ${ }^{15}$ In this scheme, an unknown two-particle state of general form, whether entangled or not, can be transmitted from a sender to a receiver with the help of partly pure entangled four-particle state with certain probability. Unfortunately in their protocol sixteen unitary transformations must be implemented. Obviously, it is not favorable to the experimental realization of teleportation. More recently in terms of only single-qubit gates, two-qubit controlled-not (CNOT) gates and measurements on single qubits, Liu et al presented quantum logic networks for probabilistic teleportation of a single qubit and a two-particle entangled state, using a partially entangled pair and a three-particle nonmaximally entangled state, respectively. ${ }^{20}$ The quantum circuit of the probabilistic teleportation is then constructed. Clearly the quantum logic networks for probabilistic teleportation will be important in realizing the teleportation scheme in the experiment. In this paper we will simplify the scheme of Ref. [15] first, then give a quantum logic network for probabilistic teleportation of two-particle state of general form.

Suppose that an unknown two-particle state of general form that will be teleported is

$$
|\phi\rangle_{12}=a|00\rangle_{12}+b|01\rangle_{12}+c|10\rangle_{12}+d|11\rangle_{12}
$$

and the quantum channel is in the following state

$$
|\phi\rangle_{3456}=\alpha|0000\rangle_{3456}+\beta|1001\rangle_{3456}+\gamma|0110\rangle_{3456}+\kappa|1111\rangle_{3456}
$$

Here $a, b, c, d$ are arbitrary complex numbers satisfying $|a|^{2}+|b|^{2}+|c|^{2}+|d|^{2}=1$, while $\alpha, \beta, \gamma$, and $\kappa$ are real, and $|\alpha|$ is smaller than others.

The particles 3 and 4 of the state $|\phi\rangle_{3456}$ and particle pair $(1,2)$ belong to the sender Alice, while other two particles 5 and 6 of the state $|\phi\rangle_{3456}$ are at the receiver Bob's side. Thus, the total state of the system is $|\Psi\rangle_{123456}=|\phi\rangle_{12}|\phi\rangle_{3456}$. If two Bell state measurements between particle 2 and particle 3 and between particle 1 and particle 4 respectively are performed by Alice, particles 5 and 6 will be collapsed into the following unnormalized states

$$
\begin{aligned}
\left|\psi_{0}\right\rangle_{56}={ }_{14}\left\langle\left.\Phi^{+}\right|_{23}\left\langle\Phi^{+} \mid \Psi\right\rangle_{123456}\right. & =\frac{1}{2}\left[a \alpha|00\rangle_{56}+b \beta|01\rangle_{56}+c \gamma|10\rangle_{56}+d \kappa|11\rangle_{56}\right], \\
\left|\psi_{1}\right\rangle_{56}={ }_{14}\left\langle\left.\Phi^{-}\right|_{23}\left\langle\Phi^{+} \mid \Psi\right\rangle_{123456}\right. & =\frac{1}{2}\left[a \alpha|00\rangle_{56}+b \beta|01\rangle_{56}-c \gamma|10\rangle_{56}-d \kappa|11\rangle_{56}\right], \\
\left|\psi_{2}\right\rangle_{56}={ }_{14}\left\langle\left.\Psi^{+}\right|_{23}\left\langle\Phi^{+} \mid \Psi\right\rangle_{123456}\right. & =\frac{1}{2}\left[c \alpha|00\rangle_{56}+d \beta|01\rangle_{56}+a \gamma|10\rangle_{56}+b \kappa|11\rangle_{56}\right],
\end{aligned}
$$




$$
\begin{aligned}
&\left|\psi_{3}\right\rangle_{56}={ }_{14}\left\langle\left.\Psi^{-}\right|_{23}\left\langle\Phi^{+} \mid \Psi\right\rangle_{123456}=\frac{1}{2}\left[-c \alpha|00\rangle_{56}-d \beta|01\rangle_{56}+a \gamma|10\rangle_{56}+b \kappa|11\rangle_{56}\right],\right. \\
&\left|\psi_{4}\right\rangle_{56}={ }_{14}\left\langle\left.\Phi^{+}\right|_{23}\left\langle\Phi^{-} \mid \Psi\right\rangle_{123456}\right.=\frac{1}{2}\left[a \alpha|00\rangle_{56}-b \beta|01\rangle_{56}+c \gamma|10\rangle_{56}-d \kappa|11\rangle_{56}\right], \\
&\left|\psi_{5}\right\rangle_{56}={ }_{14}\left\langle\left.\Phi^{-}\right|_{23}\left\langle\Phi^{-} \mid \Psi\right\rangle_{123456}\right.=\frac{1}{2}\left[a \alpha|00\rangle_{56}-b \beta|01\rangle_{56}-c \gamma|10\rangle_{56}+d \kappa|11\rangle_{56}\right], \\
&\left|\psi_{6}\right\rangle_{56}={ }_{14}\left\langle\Psi^{+}{ }_{23}\left\langle\Phi^{-} \mid \Psi\right\rangle_{123456}\right.=\frac{1}{2}\left[c \alpha|00\rangle_{56}-d \beta|01\rangle_{56}+a \gamma|10\rangle_{56}-b \kappa|11\rangle_{56}\right], \\
&\left|\psi_{7}\right\rangle_{56}={ }_{14}\left\langle\left.\Psi^{-}\right|_{23}\left\langle\Phi^{-} \mid \Psi\right\rangle_{123456}=\right. \frac{1}{2}\left[-c \alpha|00\rangle_{56}+d \beta|01\rangle_{56}+a \gamma|10\rangle_{56}-b \kappa|11\rangle_{56}\right], \\
&\left|\psi_{8}\right\rangle_{56}={ }_{14}\left\langle\left.\Phi^{+}\right|_{23}\left\langle\Psi^{+} \mid \Psi\right\rangle_{123456}=\right. \frac{1}{2}\left[b \alpha|00\rangle_{56}+a \beta|01\rangle_{56}+d \gamma|10\rangle_{56}+c \kappa|11\rangle_{56}\right], \\
&\left|\psi_{9}\right\rangle_{56}={ }_{14}\left\langle\left.\Phi^{-}\right|_{23}\left\langle\Psi^{+} \mid \Psi\right\rangle_{123456}=\right. \frac{1}{2}\left[b \alpha|00\rangle_{56}+a \beta|01\rangle_{56}-d \gamma|10\rangle_{56}-c \kappa|11\rangle_{56}\right], \\
&\left|\psi_{10}\right\rangle_{56}={ }_{14}\left\langle\left.\Psi^{+}\right|_{23}\left\langle\Psi^{+} \mid \Psi\right\rangle_{123456}=\frac{1}{2}\left[d \alpha|00\rangle_{56}+c \beta|01\rangle_{56}+b \gamma|10\rangle_{56}+a \kappa|11\rangle_{56}\right],\right. \\
&\left|\psi_{11}\right\rangle_{56}={ }_{14}\left\langle\left.\Psi^{-}\right|_{23}\left\langle\Psi^{+} \mid \Psi\right\rangle_{123456}=\frac{1}{2}\left[-d \alpha|00\rangle_{56}-c \beta|01\rangle_{56}+b \gamma|10\rangle_{56}+a \kappa|11\rangle_{56}\right],\right. \\
&\left|\psi_{12}\right\rangle_{56}={ }_{14}\left\langle\left.\Phi^{+}\right|_{23}\left\langle\Psi^{-} \mid \Psi\right\rangle_{123456}=\frac{1}{2}\left[-b \alpha|00\rangle_{56}+a \beta|01\rangle_{56}-d \gamma|10\rangle_{56}+c \kappa|11\rangle_{56}\right],\right. \\
&\left|\psi_{13}\right\rangle_{56}={ }_{14}\left\langle\left.\Phi^{-}\right|_{23}\left\langle\Psi^{-} \mid \Psi\right\rangle_{123456}=\frac{1}{2}\left[-b \alpha|00\rangle_{56}+a \beta|01\rangle_{56}+d \gamma|10\rangle_{56}-c \kappa|11\rangle_{56}\right],\right. \\
&\left|\psi_{14}\right\rangle_{56}={ }_{14}\left\langle\left.\Psi^{+}\right|_{23}\left\langle\Psi^{-} \mid \Psi\right\rangle_{123456}=\frac{1}{2}\left[-d \alpha|00\rangle_{56}+c \beta|01\rangle_{56}-b \gamma|10\rangle_{56}+a \kappa|11\rangle_{56}\right],\right. \\
& \psi_{23}\left\langle\Psi^{-} \mid \Psi\right\rangle_{123456}=\frac{1}{2}\left[d \alpha|00\rangle_{56}-c \beta|01\rangle_{56}-b \gamma|10\rangle_{56}+a \kappa|11\rangle_{56}\right] .
\end{aligned}
$$

Here

$$
\begin{aligned}
& \left|\Phi^{ \pm}\right\rangle_{23}=\frac{1}{\sqrt{2}}\left(|00\rangle_{23} \pm|11\rangle_{23}\right),\left|\Psi^{ \pm}\right\rangle_{23}=\frac{1}{\sqrt{2}}\left(|01\rangle_{23} \pm|10\rangle_{23}\right) \\
& \left|\Phi^{ \pm}\right\rangle_{14}=\frac{1}{\sqrt{2}}\left(|00\rangle_{14} \pm|11\rangle_{14}\right),\left|\Psi^{ \pm}\right\rangle_{14}=\frac{1}{\sqrt{2}}\left(|01\rangle_{14} \pm|10\rangle_{14}\right)
\end{aligned}
$$

are the Bell states of the particles 2 and 3, and particles 1 and 4 respectively.

After the Bell state measurements, Alice informs Bob of the measurement outcomes through a classical communication, then Bob understands exactly in which one of the sixteen states in Eqs. (3)-(18) is located particles 5 and 6 . Now an auxiliary particle $a$ with the initial state $|0\rangle_{a}$ is 
introduced by Bob. On the basis $\left\{|000\rangle_{56 a},|001\rangle_{56 a},|010\rangle_{56 a},|011\rangle_{56 a},|100\rangle_{56 a},|101\rangle_{56 a},|110\rangle_{56 a}\right.$, $\left.|111\rangle_{56 a}\right\}$, Bob will perform a collective unitary transformation

$$
U_{0}=\left(\begin{array}{cccccccc}
1 & 0 & 0 & 0 & 0 & 0 & 0 & 0 \\
0 & -\frac{\alpha}{\beta} & \sqrt{1-\frac{\alpha^{2}}{\beta^{2}}} & 0 & 0 & 0 & 0 & 0 \\
0 & \sqrt{1-\frac{\alpha^{2}}{\beta^{2}}} & \frac{\alpha}{\beta} & 0 & 0 & 0 & 0 & 0 \\
0 & 0 & 0 & -\frac{\alpha}{\gamma} & \sqrt{1-\frac{\alpha^{2}}{\gamma^{2}}} & 0 & 0 & 0 \\
0 & 0 & 0 & \sqrt{1-\frac{\alpha^{2}}{\gamma^{2}}} & \frac{\alpha}{\gamma} & 0 & 0 & 0 \\
0 & 0 & 0 & 0 & 0 & -1 & 0 & 0 \\
0 & 0 & 0 & 0 & 0 & 0 & \frac{\alpha}{\kappa} & \sqrt{1-\frac{\alpha^{2}}{\kappa^{2}}} \\
0 & 0 & 0 & 0 & 0 & 0 & \sqrt{1-\frac{\alpha^{2}}{\kappa^{2}}} & -\frac{\alpha}{\kappa}
\end{array}\right)
$$

on the state of particles 5,6 and $a$. After that a measurement on the auxiliary qubit $a$ is performed by Bob. If the result is $|1\rangle_{a}$, the teleportation fails. However if the measurement outcome $|0\rangle_{a}$ is obtained, the state of the particles 5 and 6 will be one of the following states

$$
\begin{aligned}
& \left|\varphi_{0}\right\rangle_{56}=a|00\rangle_{56}+b|01\rangle_{56}+c|10\rangle_{56}+d|11\rangle_{56} \\
& \left|\varphi_{1}\right\rangle_{56}=a|00\rangle_{56}+b|01\rangle_{56}-c|10\rangle_{56}-d|11\rangle_{56}, \\
& \left|\varphi_{2}\right\rangle_{56}=c|00\rangle_{56}+d|01\rangle_{56}+a|10\rangle_{56}+b|11\rangle_{56}, \\
& \left|\varphi_{3}\right\rangle_{56}=-c|00\rangle_{56}-d|01\rangle_{56}+a|10\rangle_{56}+b|11\rangle_{56} \\
& \left|\varphi_{4}\right\rangle_{56}=a|00\rangle_{56}-b|01\rangle_{56}+c|10\rangle_{56}-d|11\rangle_{56} \\
& \left|\varphi_{5}\right\rangle_{56}=a|00\rangle_{56}-b|01\rangle_{56}-c|10\rangle_{56}+d|11\rangle_{56} \\
& \left|\varphi_{6}\right\rangle_{56}=c|00\rangle_{56}-d|01\rangle_{56}+a|10\rangle_{56}-b|11\rangle_{56} \\
& \left|\varphi_{7}\right\rangle_{56}=-c|00\rangle_{56}+d|01\rangle_{56}+a|10\rangle_{56}-b|11\rangle_{56} \\
& \left|\varphi_{8}\right\rangle_{56}=b|00\rangle_{56}+a|01\rangle_{56}+d|10\rangle_{56}+c|11\rangle_{56} \\
& \left|\varphi_{9}\right\rangle_{56}=b|00\rangle_{56}+a|01\rangle_{56}-d|10\rangle_{56}-c|11\rangle_{56} \\
& \left|\varphi_{10}\right\rangle_{56}=d|00\rangle_{56}+c|01\rangle_{56}+b|10\rangle_{56}+a|11\rangle_{56} \\
& \left|\varphi_{11}\right\rangle_{56}=-d|00\rangle_{56}-c|01\rangle_{56}+b|10\rangle_{56}+a|11\rangle_{56} \\
& \left|\varphi_{12}\right\rangle_{56}=-b|00\rangle_{56}+a|01\rangle_{56}-d|10\rangle_{56}+c|11\rangle_{56} \\
& \left|\varphi_{13}\right\rangle_{56}=-b|00\rangle_{56}+a|01\rangle_{56}+d|10\rangle_{56}-c|11\rangle_{56}
\end{aligned}
$$




$$
\begin{aligned}
& \left|\varphi_{14}\right\rangle_{56}=-d|00\rangle_{56}+c|01\rangle_{56}-b|10\rangle_{56}+a|11\rangle_{56} \\
& \left|\varphi_{15}\right\rangle_{56}=d|00\rangle_{56}-c|01\rangle_{56}-b|10\rangle_{56}+a|11\rangle_{56}
\end{aligned}
$$

according to the outcomes of Bell state measurements. The teleportation can be successfully achieved with the classical information from Alice and a corresponding unitary operation which is easy designed on the particles 5 and 6 . For example we can transform $\left|\varphi_{12}\right\rangle_{56}=-b|00\rangle_{56}+$ $a|01\rangle_{56}-d|10\rangle_{56}+c|11\rangle_{56}$ into the state $a|00\rangle_{56}+b|01\rangle_{56}+c|10\rangle_{56}+d|11\rangle_{56}$ by using $\sigma_{z} \sigma_{x}$ on the particle 6 , where $\sigma_{x}, \sigma_{z}$ are Pauli operators. In this new scheme we only use one unitary transformation $U_{0}$, which can carry out the task with the same probability as $U_{0}, U_{1}, \ldots, U_{15}$ of Ref. [15]. This will make the teleportation easily realized.

Evidently the implementation of $U_{0}$ plays an essential role in the teleportation of two-particle state of general form. Barenco et al showed that a set of gates consisting of all one-bit quantum gates and the two-bit controlled-not (CNOT) gates is universal in the sense that all unitary operation on arbitrarily many bits can be expressed as compositions of these gates. They investigated a general simulation of a three-bit controlled-u gate for an arbitrary one-bit unitary operation $u$ using only these basic gates. ${ }^{21}$ It is tedious but straightforward to prove that $U_{0}$ can be expressed as

$$
\begin{aligned}
U_{0}= & \left(I \otimes \Lambda_{2}(X)\right) C_{13}\left(I \otimes \Lambda_{1}(X)\right) C_{12}\left(I \otimes \Lambda_{2}(X)\right) C_{13}(I \otimes X \otimes I)\left(\Lambda_{1}(X) \otimes I\right)\left(I \otimes \Lambda_{2}(X)\right) C_{13} \\
& \left(I \otimes \Lambda_{2}(X)\right) C_{13}\left(I \otimes \Lambda_{1}(X)\right) C_{12}\left(I \otimes \Lambda_{2}(X)\right) C_{13}(I \otimes I \otimes X)\left(I \otimes \Lambda_{1}(X)\right) C_{23} C_{13} \\
& \left(I \otimes \Lambda_{2}(X)\right) C_{13}\left(I \otimes \Lambda_{1}(X)\right) C_{12}\left(I \otimes \Lambda_{2}(X)\right) C_{13}(I \otimes X \otimes I)\left(\Lambda_{1}(X) \otimes I\right) \\
& \left(I \otimes \Lambda_{2}(X)\right) C_{13}\left(I \otimes \Lambda_{2}(X)\right) C_{13}\left(I \otimes \Lambda_{1}(X)\right) C_{12}\left(I \otimes \Lambda_{2}(X)\right) C_{13}(I \otimes I \otimes X) \\
& \left(I \otimes \Lambda_{1}(X)\right) C_{13} C_{23}\left(I \otimes \Lambda_{1}(X)\right) C_{12}\left(I \otimes \Lambda_{2}(X)\right) C_{13}\left(I \otimes \Lambda_{1}(X)\right) C_{12}\left(I \otimes \Lambda_{2}(X)\right) \\
& C_{13} C_{12}\left(\Lambda_{2}(X) \otimes I\right) C_{23} C_{12} \Lambda_{23}\left(u_{1}\right) C_{12}\left(\Lambda_{2}(X) \otimes I\right) C_{23} C_{12} C_{13}\left(I \otimes \Lambda_{2}(X)\right) C_{13} \\
& \left(I \otimes \Lambda_{1}(X)\right) C_{12}\left(I \otimes \Lambda_{2}(X)\right) C_{13}(I \otimes X \otimes I)\left(\Lambda_{1}(X) \otimes I\right)\left(I \otimes \Lambda_{2}(X)\right) C_{13} \\
& \left(I \otimes \Lambda_{2}(X)\right) C_{13}\left(I \otimes \Lambda_{1}(X)\right) C_{12}\left(I \otimes \Lambda_{2}(X)\right) C_{13}(I \otimes I \otimes X)\left(I \otimes \Lambda_{1}(X)\right) C_{13} \Lambda_{13}\left(u_{2}\right) \\
& C_{13}\left(I \otimes \Lambda_{2}(X)\right) C_{13}\left(I \otimes \Lambda_{1}(X)\right) C_{12}\left(I \otimes \Lambda_{2}(X)\right) C_{13}(I \otimes X \otimes I)\left(\Lambda_{1}(X) \otimes I\right)\left(I \otimes \Lambda_{2}(X)\right) \\
& C_{13}\left(I \otimes \Lambda_{2}(X)\right) C_{13}\left(I \otimes \Lambda_{1}(X)\right) C_{12}\left(I \otimes \Lambda_{2}(X)\right) C_{13}(I \otimes I \otimes X)\left(I \otimes \Lambda_{1}(X)\right) C_{13} \Lambda_{12}\left(u_{3}\right) \\
& (I \otimes I \otimes Z)\left(I \otimes \Lambda_{2}(X)\right) C_{13}\left(I \otimes \Lambda_{1}(X)\right) C_{12}\left(I \otimes \Lambda_{2}(X)\right) C_{13}\left(I \otimes \Lambda_{1}(X)\right) C_{12} C_{23} C_{13} \\
& \left(I \otimes \Lambda_{2}(X)\right) C_{13}\left(I \otimes \Lambda_{1}(X)\right) C_{12}\left(I \otimes \Lambda_{2}(X)\right) C_{13}(I \otimes X \otimes I)\left(\Lambda_{1}(X) \otimes I\right)\left(I \otimes \Lambda_{2}(X)\right) C_{13} \\
& \left(I \otimes \Lambda_{2}(X)\right) C_{13}\left(I \otimes \Lambda_{1}(X)\right) C_{12}\left(I \otimes \Lambda_{2}(X)\right) C_{13}(I \otimes I \otimes X)\left(I \otimes \Lambda_{1}(X)\right) C_{13} C_{23} \\
& \left(I \otimes \Lambda_{2}(X)\right) C_{13}\left(I \otimes \Lambda_{1}(X)\right) C_{12}\left(I \otimes \Lambda_{2}(X)\right) C_{13}(I \otimes X \otimes I)\left(\Lambda_{1}(X) \otimes I\right)\left(I \otimes \Lambda_{2}(X)\right) \\
& C_{13}\left(I \otimes \Lambda_{2}(X)\right) C_{13}\left(I \otimes \Lambda_{1}(X)\right) C_{12}\left(I \otimes \Lambda_{2}(X)\right) C_{13}(I \otimes I \otimes X)\left(I \otimes \Lambda_{1}(X)\right)
\end{aligned}
$$


Here $\Lambda_{i j}(u)$ are the three-bit controlled-u gates, $i, j=1,2,3$, and $\Lambda_{i}(X)$ are controlled-NOT gates, $i=1,2$;

$$
\begin{aligned}
& I=\left(\begin{array}{cc}
1 & 0 \\
0 & 1
\end{array}\right) ; \quad X=\left(\begin{array}{cc}
0 & 1 \\
1 & 0
\end{array}\right) ; \quad Z=\left(\begin{array}{cc}
1 & 0 \\
0 & -1
\end{array}\right) \\
& \Lambda_{1}(X)=\left(\begin{array}{cccc}
1 & 0 & 0 & 0 \\
0 & 1 & 0 & 0 \\
0 & 0 & 0 & 1 \\
0 & 0 & 1 & 0
\end{array}\right) ; \quad \Lambda_{2}(X)=\left(\begin{array}{cccc}
1 & 0 & 0 & 0 \\
0 & 0 & 0 & 1 \\
0 & 0 & 1 & 0 \\
0 & 1 & 0 & 0
\end{array}\right) \\
& \Lambda_{23}\left(u_{1}\right)=\left(\begin{array}{cccccccc}
1 & 0 & 0 & 0 & 0 & 0 & 0 & 0 \\
0 & 1 & 0 & 0 & 0 & 0 & 0 & 0 \\
0 & 0 & 1 & 0 & 0 & 0 & 0 & 0 \\
0 & 0 & 0 & -\frac{\alpha}{\beta} & 0 & 0 & 0 & \sqrt{1-\frac{\alpha^{2}}{\beta^{2}}} \\
0 & 0 & 0 & 0 & 1 & 0 & 0 & 0 \\
0 & 0 & 0 & 0 & 0 & 1 & 0 & 0 \\
0 & 0 & 0 & 0 & 0 & 0 & 1 & 0 \\
0 & 0 & 0 & -\sqrt{1-\frac{\alpha^{2}}{\beta^{2}}} & 0 & 0 & 0 & -\frac{\alpha}{\beta}
\end{array}\right)
\end{aligned}
$$

$$
\Lambda_{13}\left(u_{2}\right)=\left(\begin{array}{cccccccc}
1 & 0 & 0 & 0 & 0 & 0 & 0 & 0 \\
0 & 1 & 0 & 0 & 0 & 0 & 0 & 0 \\
0 & 0 & 1 & 0 & 0 & 0 & 0 & 0 \\
0 & 0 & 0 & 1 & 0 & 0 & 0 & 0 \\
0 & 0 & 0 & 0 & 1 & 0 & 0 & 0 \\
0 & 0 & 0 & 0 & 0 & -\frac{\alpha}{\gamma} & 0 & \sqrt{1-\frac{\alpha^{2}}{\gamma^{2}}} \\
0 & 0 & 0 & 0 & 0 & 0 & 1 & 0 \\
0 & 0 & 0 & 0 & 0 & -\sqrt{1-\frac{\alpha^{2}}{\gamma^{2}}} & 0 & -\frac{\alpha}{\gamma}
\end{array}\right) ;
$$




$$
\Lambda_{12}\left(u_{3}\right)=\left(\begin{array}{cccccccc}
1 & 0 & 0 & 0 & 0 & 0 & 0 & 0 \\
0 & 1 & 0 & 0 & 0 & 0 & 0 & 0 \\
0 & 0 & 1 & 0 & 0 & 0 & 0 & 0 \\
0 & 0 & 0 & 1 & 0 & 0 & 0 & 0 \\
0 & 0 & 0 & 0 & 1 & 0 & 0 & 0 \\
0 & 0 & 0 & 0 & 0 & 1 & 0 & 0 \\
0 & 0 & 0 & 0 & 0 & 0 & \frac{\alpha}{\kappa} & -\sqrt{1-\frac{\alpha^{2}}{\kappa^{2}}} \\
0 & 0 & 0 & 0 & 0 & 0 & \sqrt{1-\frac{\alpha^{2}}{\kappa^{2}}} & \frac{\alpha}{\kappa}
\end{array}\right) ;
$$

$$
\Lambda_{12}(X)=C_{12}=\left(\begin{array}{cccccccc}
1 & 0 & 0 & 0 & 0 & 0 & 0 & 0 \\
0 & 1 & 0 & 0 & 0 & 0 & 0 & 0 \\
0 & 0 & 1 & 0 & 0 & 0 & 0 & 0 \\
0 & 0 & 0 & 1 & 0 & 0 & 0 & 0 \\
0 & 0 & 0 & 0 & 1 & 0 & 0 & 0 \\
0 & 0 & 0 & 0 & 0 & 1 & 0 & 0 \\
0 & 0 & 0 & 0 & 0 & 0 & 0 & 1 \\
0 & 0 & 0 & 0 & 0 & 0 & 1 & 0
\end{array}\right)
$$

$$
\Lambda_{23}(X)=C_{23}=\left(\begin{array}{cccccccc}
1 & 0 & 0 & 0 & 0 & 0 & 0 & 0 \\
0 & 1 & 0 & 0 & 0 & 0 & 0 & 0 \\
0 & 0 & 1 & 0 & 0 & 0 & 0 & 0 \\
0 & 0 & 0 & 0 & 0 & 0 & 0 & 1 \\
0 & 0 & 0 & 0 & 1 & 0 & 0 & 0 \\
0 & 0 & 0 & 0 & 0 & 1 & 0 & 0 \\
0 & 0 & 0 & 0 & 0 & 0 & 1 & 0 \\
0 & 0 & 0 & 1 & 0 & 0 & 0 & 0
\end{array}\right)
$$




$$
\Lambda_{13}(X)=C_{13}=\left(\begin{array}{cccccccc}
1 & 0 & 0 & 0 & 0 & 0 & 0 & 0 \\
0 & 1 & 0 & 0 & 0 & 0 & 0 & 0 \\
0 & 0 & 1 & 0 & 0 & 0 & 0 & 0 \\
0 & 0 & 0 & 1 & 0 & 0 & 0 & 0 \\
0 & 0 & 0 & 0 & 1 & 0 & 0 & 0 \\
0 & 0 & 0 & 0 & 0 & 0 & 0 & 1 \\
0 & 0 & 0 & 0 & 0 & 0 & 1 & 0 \\
0 & 0 & 0 & 0 & 0 & 1 & 0 & 0
\end{array}\right) .
$$

Combining the results obtained by Barenco et al and the Eq.(36) we see that single qubit and CNOT gates can be used to implement unitary operation $U_{0}$ and can easily figure out the quantum gate array of the unitary operation $U_{0}$. For the sake of saving space we will not depict it out.

Based on the simplification scheme and the decomposition of unitary operator $U_{0}$ we can constructed a quantum network for probabilistic teleportation of an unknown two-particle state of general form. It is shown in Fig. 1.

Here $R, R^{\prime}, R^{\prime \prime}$ are single-qubit rotation transformations, and $H$ is a Hadamard gate defined as $H=\frac{1}{\sqrt{2}}\left(\begin{array}{cc}1 & 1 \\ 1 & -1\end{array}\right)$. We carefully choose $R, R^{\prime}$, and $R^{\prime \prime}$ to make the state of the particles 3,4 , 5 , and 6 to be the quantum channel $|\phi\rangle_{3456}=\alpha|0000\rangle_{3456}+\beta|1001\rangle_{3456}+\gamma|0110\rangle_{3456}+\kappa|1111\rangle_{3456}$ by the first dash line. If the measurement result on the auxiliary qubit at the output state is $|0\rangle_{a}$, the teleportation is successful with the final state of the particle 5 and 6 being reconstructed as the initial state to be teleported. From Fig.1, we see that the probabilistic teleportation can be divided into two steps. The first step is a purification process in which Bob can concentrate the state $\alpha|0000\rangle_{3456}+\beta|0110\rangle_{3456}+\gamma|1001\rangle_{3456}+\kappa|1111\rangle_{3456}$ into a maximally entangled state by introducing an auxiliary particle $a$ and performing a collective unitary operation $U_{0}$. The second step is the standard teleportation using the maximal entanglement as the quantum channel.

Note that a controlled unitary operation acting on any number of qubits followed by the measurement of the control qubit can be replaced by the measurement of the control qubit preceding the controlled operation. ${ }^{20}$ Therefore, Fig.1 can be re-expressed as Fig.2.

In Fig.2, the controlled operation can be realized locally by Bob depending on the results of the four measurements Alice performed on her own qubit. If and only if the outcome of 
Alice's measurement is 1 , Bob can execute the controlled- $R_{x}$ or controlled- $R_{z}$, where $R_{x}=X$, $R_{z}=H R_{x} H$. If the measurement result of the auxiliary particle is $|1\rangle_{a}$, the teleportation fails. If the result is $|0\rangle_{a}$, the final state of particles 5 and 6 at Bob's side will be collapsed into $|\phi\rangle_{12}=$ $a|00\rangle_{12}+b|01\rangle_{12}+c|10\rangle_{12}+d|11\rangle_{12}$, which is the desired state. That is to say, if the measurement outcome on the state of the auxiliary qubit is $|0\rangle_{a}$, perfect teleportation is accomplished.

In summary, a simplification scheme for probabilistic teleporting an unknown two-particle state of general form is given. By means of the primitive operations consisting of single-qubit gates, twoqubit controlled-not gates, Von Neumann measurement and classically controlled operations, we construct an efficient quantum logical network for implementing the new scheme of probabilistic teleportation of a two-particle state of general form. Both the product state and entangled state of two-particles can be transmitted from the sender to receiver by the partly pure entangled fourparticle state with certain probability. We hope that this scheme will be realized by experiment.

\section{REFERENCES}

1 C. H. Bennett et al, Phys. Rev. Lett. 70 (1993) 1895.

2 J. I. Cirac and P. Zoller, Phys. Rev. Lett. 74 (1995) 4091.

3 A. Barenco et al, Phys. Rev. Lett. 74 (1995)4083.

4 A. K. Ekert, Phys. Rev. Lett. 67 (1991) 661.

${ }^{5}$ C. H. Bennett, Phys. Rev. Lett. 68 (1992) 3121.

6 Y. Zhang et al, Chin. Phys. Lett. 15 (1998) 238.

7 B. S. Shi and G. C. Guo, Chin. Phys. Lett. 14 (1997) 521.

${ }^{8}$ C. H. Bennett and S. J. Wiesner, Phys. Rev. Lett. 69 (1992) 2881.

9 M. Ikram, S. Y. Zhu and M. S. Zubairy, Phys. Rev. A62 (2000) 022307.

10 W. L. Li, C. F. Li and G. C. Guo, Phys. Rev. A61 (2000) 034301.

11 V. N. Gorbachev and A. I. Trubilko, J. Exp. Theor. Phys. 91 (2000) 894.

12 H. Lu and G. C. Guo, Phys. Lett. A276 (2000) 209.

13 B. Zeng, X. S. Liu, Y. S. Li and G. L. Long, Commun. Theor. Phys. 38 (2002) 537.

14 B. S. Shi et al, Phys. Lett. A268 (2000) 161.

15 F. L. Yan, H. G. Tan and L. G. Yang, Commun. Theor. Phys. 37 (2002) 649.

16 D. Bouwmeester et al, Nature 390 (1997) 575.

17 A. Furusawa et al, Science 282 (1998) 706.

18 D. Boschi et al, Phys. Rev. Lett. 80 (1998) 1121.

19 M. A. Nielsen et al, Nature 396 (1998) 52.

20 J. M. Liu, Y. S. Zhang and G. C. Guo, Chinese Physics 12 (2003) 251.

21 A. Barenco et al, Phys. Rev. A 52 (1995) 3457. 


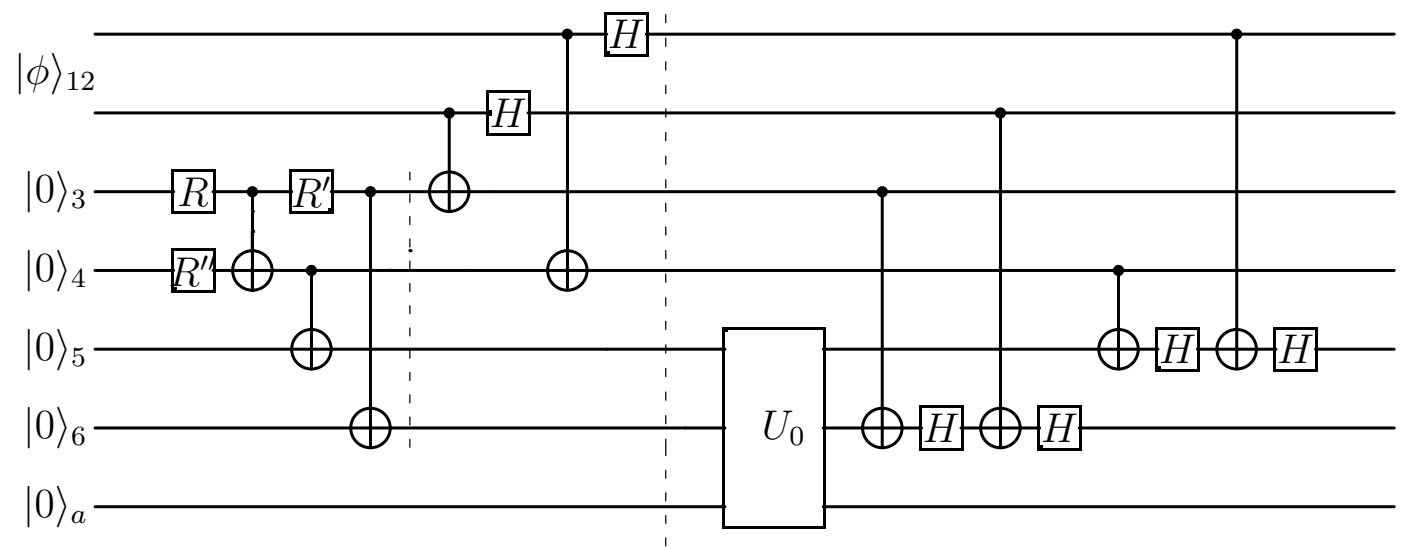

Figure 1: Quantum circuit for probabilistic teleportation of a two-particle state of general form.

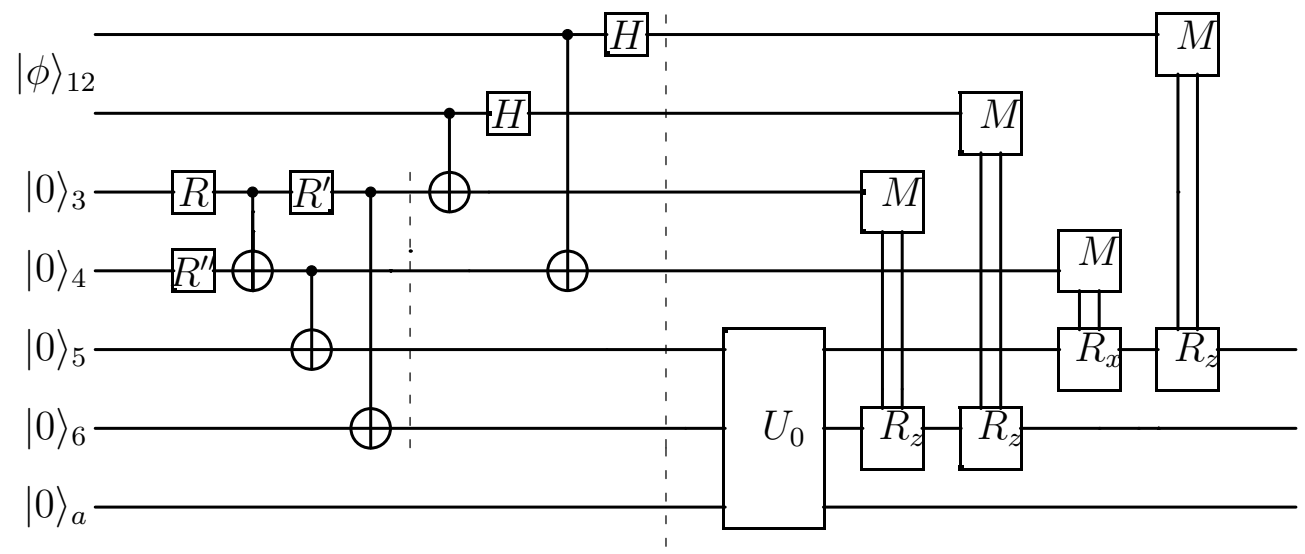

Figure 2: The same as Fig.1, but Von Neumann measurement $M$ and classically controlled operation are made at the end instead. 